\title{
Sinusoidal Gratings for Optimized Light Management in c-Si Thin-Film Solar Cells
}

\author{
Klaus Jäger ${ }^{a b}$ Grit Köppel, ${ }^{a}$ Carlo Barth, ${ }^{a b}$ Martin Hammerschmidt, ${ }^{b}$ Sven Herrmann, ${ }^{b}$ Sven \\ Burger, ${ }^{b c}$ Frank Schmidt, ${ }^{b c}$ and Christiane Becker ${ }^{a}$ \\ ${ }^{a}$ Young Investigator Group Nano-SIPPE, Helmholtz-Zentrum Berlin für Materialien und \\ Energie GmbH, Kekuléstr. 5, 12489 Berlin, Germany; \\ ${ }^{b}$ Zuse Institute Berlin, Takustr. 7, 14195 Berlin, Germany; \\ ${ }^{c}$ JCMwave GmbH, Bolivarallee 22, 14050 Berlin, Germany
}

\begin{abstract}
Hexagonal sinusoidal nanotextures are well suited to couple light into silicon on glass at normal incidence, as we have shown in an earlier publication [K. Jäger et al., Opt. Express 24, A569 (2016)]. In this manuscript we discuss how these nanotextures perform under oblique incidence illumination. For this numerical study we use a rigorous solver for the Maxwell equations. We discuss nanotextures with periods between $350 \mathrm{~nm}$ and $730 \mathrm{~nm}$ and an aspect ratio of 0.5 .
\end{abstract}

Keywords: Diffraction gratings, antireflection coatings, numerical approximation and analysis, solar energy

\section{INTRODUCTION}

Liquid-phase crystallized silicon (LPC-Si) thin-film solar cells have the potential to combine the high efficiency achieved with crystalline silicon (c-Si) with the advantages of thin-film technology. For making the LPC-Si films, an about $10 \mu \mathrm{m}$ thick nanocrystalline Si film is deposited onto a glass substrate and subsequently crystallized using a scanning electron or laser beam. ${ }^{1-4}$

As the LPC-Si absorber is only about $10 \mu \mathrm{m}$ thick, advanced light management techniques are required to maximize the absorption. Light trapping enhances absorption at long wavelengths, where $\mathrm{Si}$ - an indirect bandgap material - absorbs only poorly. Further, anti-reflective (AR) textures are essential to maximize the fraction of the incident light coupled into the silicon absorber.

Because the light is incident from the glass side, the anti-reflective texture has to be created on the glasssilicon interface prior to the silicon deposition and hence prior to the LPC process, when the Si is melted. As a consequence, AR textures must be rather smooth as too pronounced textures have shown to have a detrimental effect on the electrical properties of the solar cells. ${ }^{4,5}$

In an earlier contribution we extensively discussed the use of smooth hexagonal sinusoidal nanotextures with periods ranging from 350 to $1050 \mathrm{~nm}$ for effective coupling of the incident light into a silicon absorber. ${ }^{6}$ We discovered a great variability of these structures - even if the period and aspect ratio are kept constant. There, we mainly discussed results for normal incidence illumination. Figure 1(a) shows an atomic force microscopy picture of an experimentally realized hexagonal sinusoidal grating on sol-gel with $750 \mathrm{~nm}$ period prepared prepared using nanoimprint lithography. ${ }^{7}$

In this contribution, we numerically study how hexagonal sinusoidal nanotextures perform under oblique incidence. In particular, we investigate whether the trends observed for normal incidence also hold under oblique incidence. We will discuss numerical results for a simple layer stack consisting of glass, nanotextured sol-gel and Si. The presented results are obtained with electromagnetic field simulations using a finite element method.

Further author information: (Send correspondence to K.J.)

K.J.: E-mail: klaus.jaeger@helmholtz-berlin.de, Telephone: +49 (0)30 8062-41366

Ch.B.: E-mail: christiane.becker@helmholtz-berlin.de, Telephone: +49 (0)30 8062-41392 
(a)

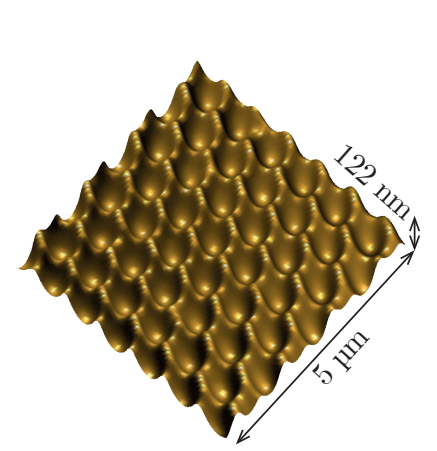

(b)

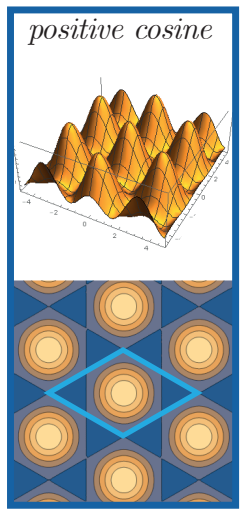

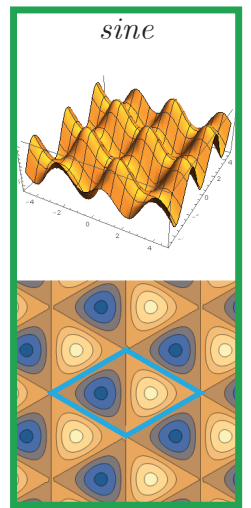

texture height

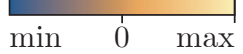

(c)
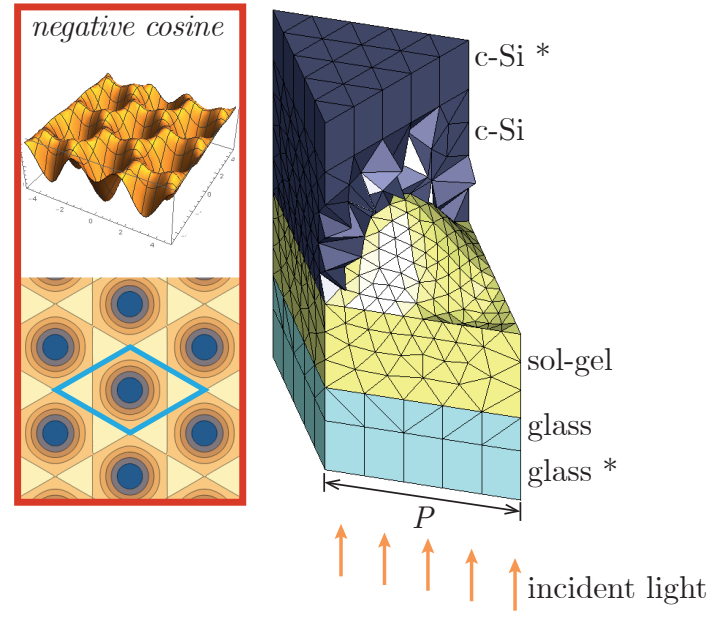

*transparent boundary

Figure 1. (a) Atomic force microscopy picture of a hexagonal sinusoidal grating on sol-gel with 750 nm period prepared using nanoimprint lithography. ${ }^{7}$ (b) Three examples of different hexagonal sinusoidal gratings, which we call positive cosine ("+cos"), sine ("sin") and negative cosine ("-cos"). The unit cell borders used for the simulations are depicted in the contour plot. (c) Visualization of the tetrahedral mesh discretizing one of the layer stacks ("+cos") used for the simulations. The light is incident from the glass side. On top and bottom, transparent boundary conditions are used.

\section{METHODS}

Figure 1(b) shows the three different sinusoidal hexagonal nanotextures investigated in this work. As explained in detail in Ref. 6 , such nanotextures are mathematically described by

$$
f_{\text {hex }}(x, y)=\cos \left[\frac{1}{2}(x+\sqrt{3} y)\right] \cos \left[\frac{1}{2}(x-\sqrt{3} y)\right] \cos (x+\phi),
$$

where $\phi$ is a structure phase that strongly affects the morphology of the nanotexture: for $\phi=0$ we have what we call a positive cosine ("+cos") texture, for $\phi=\pi / 2$ a sine ("sin") texture, and for $\phi=\pi / 2$ a negative cosine ("-cos") texture. To generate a texture with a predefined period $P$ and structure height $h$ the texture obtained using Eq. (1) has to be stretched vertically and laterally. ${ }^{6}$

The results presented in this document were obtained by solving the Maxwell equations on the 3D unit cell of the grating with appropriate source, material and boundary condition settings, using a time-harmonic finiteelement solver (JCMsuite). ${ }^{8}$ As illustrated in Fig. 1(c), the layer stacks used in the simulations are built from a tetrahedral mesh. As a result of a convergence study we found the optimal parameter settings for reaching a sufficient accuracy: the mesh side length constraint of the nanotextured sinusoidal interface is set to $50 \mathrm{~nm}$ and the maximal edge length of the tetrahedra is $100 \mathrm{~nm}$. Polynomial degrees between 2 and 4 are employed using the hp-FEM method. ${ }^{9}$

In this study we are interested in how well the different sinusoidal nanotextures couple light from the glass substrate into the Si absorber. Therefore, we assume the Si absorber to be infinitely thick, which allows us to neglect all the effects that would arise from light that is reflected back from the rear surface of the Si absorber to the front of the device. To keep the calculation time of the single simulations short, both the glass layer and the Si layer (above the nanostructure) are kept very thin at $100 \mathrm{~nm}$ and $200 \mathrm{~nm}$, respectively. In order to treat them as infinite half spaces, they are covered by perfectly matched layers that act as transparent boundaries, which are indicated in Fig. 1(c). Because of the lateral periodicity of the layers stack the other sides of the unit cell are treated as periodic boundaries.

The complex refractive index data for sol-gel were obtained with ellipsometry. We found that it does not absorb at wavelengths longer than $340 \mathrm{~nm}$. Refractive index data for crystalline $\mathrm{Si}$ and glass were provided by Helmholtz-Zentrum Berlin. In the simulations, glass is considered to be non-absorptive, hence the imaginary part of its refractive index is equal to zero. 
(a)

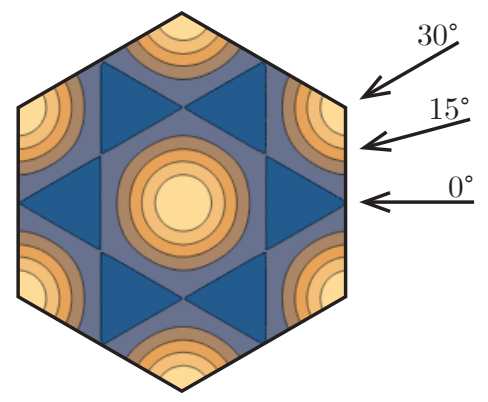

(b)

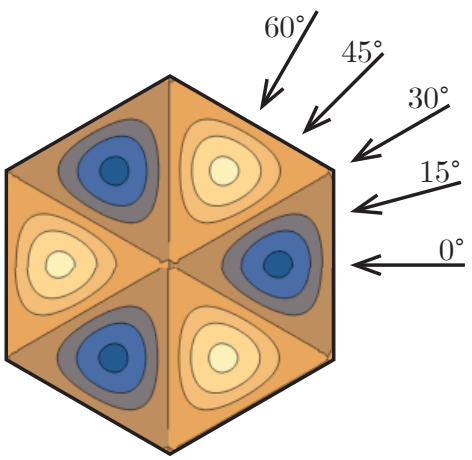

Figure 2. For averaging across all azimuth angles $\psi$ in steps of $15 \mathrm{deg}$, only a few values need to be simulated because of symmetry reasons. (a) For the "+cos" and " $-\cos$ " textures, values of $\psi=0^{\circ}, 15^{\circ}$ and $30^{\circ}$ are sufficient. (b) For the "sin" texture, also values of $\psi=45^{\circ}$ and $60^{\circ}$ must be simulated.

To analyze the results, we look at the spectra of the fraction of light that is coupled into the absorber. As a figure of merit we use the maximum achievable photocurrent density $J_{\mathrm{ph}}^{\max }$, which is the short-circuit current density that would be achieved if all the light coupled into the absorber would lead to the generation of electronhole pairs under illumination with the AM1.5 solar spectrum. ${ }^{10}$ Note that in this study the absorber is treated as infinitely thick. More details on the numerical determination of those two quantities are given in Ref. 6 .

When studying oblique incidence, also the azimuth $\psi$ of the incident wave has to be taken into account. We have chosen to average across all azimuths with steps of $15^{\circ}$. Because of the high symmetry of sinusoidal hexagonal gratings, ${ }^{11,12}$ we only need to simulate a very limited number of values of $\psi$. As illustrated in Fig. $2(\mathrm{a})$, the "+cos" and "-cos" textures exhibit $C_{6}$ symmetries, i.e. rotational symmetries of $60^{\circ}$. Hence, azimuth angles of $\psi=0^{\circ}, 15^{\circ}, 30^{\circ}$ and $45^{\circ}$ (or $-15^{\circ}$, which is equivalent) need to be simulated. Further, $-15^{\circ}$ can be omitted because it is equivalent to $+15^{\circ}$ because of the reflectional $\sigma_{y}$ symmetry with $\psi=0^{\circ}$ as the reflection axis. To calculate the average for all azimuths, a weighted average is taken where $\psi=0^{\circ}$ and $30^{\circ}$ account for $25 \%$ each and $\psi=15^{\circ}$ accounts for $50 \%$.

In contrast, the "sin" texture has a $C_{3}$ symmetry, i.e. a rotational symmetry of $120^{\circ}$, as shown in Fig. 2(b). Therefore, also $\psi=45^{\circ}$ and $60^{\circ}$ must be simulated. To average for all azimuths, a weighted average is taken where $\psi=0^{\circ}$ and $60^{\circ}$ account for $12.5 \%$ each, and $\psi=15^{\circ}, 30^{\circ}$ and $45^{\circ}$ account for $25 \%$ each.

\section{SUMMARY OF PREVIOUS RESULTS ON NORMAL INCIDENCE ILLUMINATION}

Figure 3 reviews the most important results for normal incidence. ${ }^{6}$ For all the results shown in this figure, the reflection of about $4 \%$ occurring at real devices with a flat air-glass interface was subtracted after the simulation. Further, all the results show the average of the two polarizations.

In Fig. 3(a), the maximum achievable current density is shown for the three geometries introduced in Fig. 1(b). For all geometries, a constant aspect ratio of $h / P=0.5$ was used. We see that the optimal period for in-coupling is around 500-600 nm, irrespective of the geometry. Further, the $J_{\mathrm{ph}}^{\max }$ achieved with the "-cos" texture exceeds the one achieved with the other textures for all periods.

The fraction of in-coupled light along the relevant wavelength range is shown in Fig. 3(b) for the three morphologies and a period of $P=500 \mathrm{~nm}$. We observe that the "-cos" texture outperforms the other textures for all wavelengths. In particular, the descending slope for wavelengths longer than $650 \mathrm{~nm}$ is lowest for the "-cos". For all three spectra we observe three distinctive peaks at about $330 \mathrm{~nm}, 380 \mathrm{~nm}$, and $650 \mathrm{~nm}$. These peaks arise from the lattice constant of the sinusoidal grating as we discussed in Ref. 6 . 
(a)

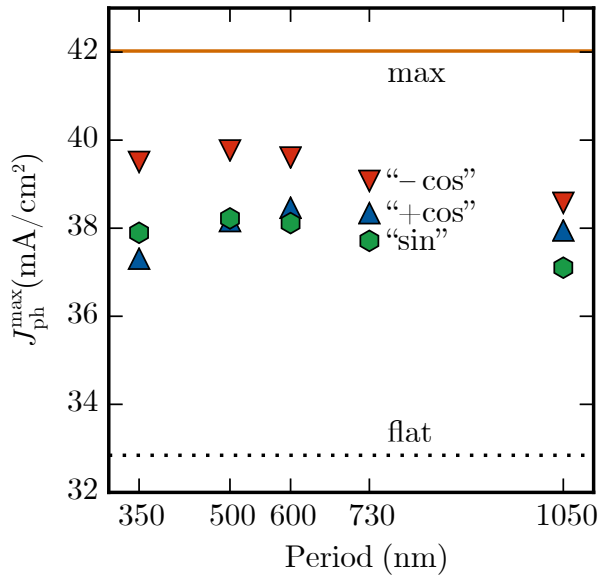

(b)

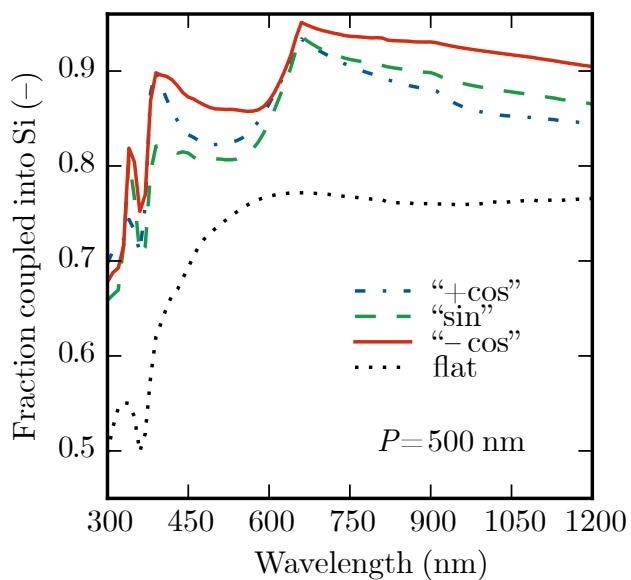

Figure 3. Simulation results for normal incidence for the layer stack illustrated in Fig. 1(c) for the three different geometries presented in Fig. 1(b) and a constant aspect ratio of $a=h / P=0.5$. (a) The maximum achievable current density $J_{\mathrm{ph}}^{\max }$ for five different periods and (b) the spectra of the in-coupled light for the three hexagonal structures and $P=500 \mathrm{~nm}$ are shown. A flat reference and, in (a), the current achieved with full absorption are shown as well. All results are corrected for the reflection loss at the air-glass interface (about 4\%). (Adapted from Ref. 6)

\section{RESULTS FOR OBLIQUE INCIDENCE}

In this section we investigate how the different sinusoidal gratings perform under oblique incidence. As parameter we use the angle of incidence of light in the glass layer, $\theta_{\text {glass }}$. In contrast to normal incidence, here, we do not take the reflection loss at the air-glass interface into account. According to Snell's law, the maximum angle $\theta_{\text {glass }}$ for light incident via a flat air-glass interface would be $41^{\circ}$. However, with (nano)structured anti-reflective coatings between air and glass, light also can be scattered into angles $>41^{\circ}$.

In Figs. 4 and 5 we present results for the three geometries with $P=500 \mathrm{~nm}$ and $h=250 \mathrm{~nm}$ and a flat layer stack for TE- and TM-polarized light, respectively. Figures 4(a) and 5(a) show the maximum achievable photocurrent densities multiplied with $1 / \cos \theta_{\text {glass }}$, where we take into account that the AM1.5 spectrum is reduced by this cosine factor for oblique incidence. As a reference, we plot the current density achieved when all the light would be coupled into the Si absorber. Figures $4(\mathrm{~b}-\mathrm{d})$ and $5(\mathrm{~b}-\mathrm{d})$ show the spectra of the fraction of light coupled into the Si for angles of $\theta_{\text {glass }}=15^{\circ}, 45^{\circ}$ and $75^{\circ}$.

For TE polarization (Fig. 4), the corrected maximum achievable current density $J_{\mathrm{ph}}^{\max } / \cos \theta_{\text {glass }}$ decreases with $\cos \theta_{\text {glass }}$. For all the angles of incidence, the negative cosine texture performs best, however, for $\cos \theta_{\text {glass }}=75^{\circ}$ only a small fraction is coupled into the absorber. The trend looks very different for TM polarization (Fig. 5). $J_{\mathrm{ph}}^{\max } / \cos \theta_{\text {glass }}$ increases until about $\theta_{\text {glass }}=60^{\circ}$, which is typical for TM polarized light, whose reflection completely vanishes at the Brewster angle. Then $J_{\mathrm{ph}}^{\max } / \cos \theta_{\text {glass }}$ decreases drastically until $75^{\circ}$. Also the spectra presented in Fig. 5(b-d) show interesting behaviour: for $\theta_{\text {glass }}=15^{\circ}$ the " - cos" performs best and the flat stack performs worst. For $\theta_{\text {glass }}=45^{\circ}$, all four samples perform very similar and for $\theta_{\text {glass }}=75^{\circ}$, the trend is just the other way round: flat performs best and "-cos" performs worst.

The dramatic decrease of the in-coupled fraction of light at $\theta_{\text {glass }}=75^{\circ}$ is caused by the contrast between the refractive indices of sol-gel and glass: for example, at $600 \mathrm{~nm} n_{\text {glass }}=1.51$ and $n_{\text {glass }}=1.44$. While this small difference has only very little effect for normal incidence, reflection at the glass-sol-gel interface gets significant for larger angles. At $\theta_{\text {glass }}=75^{\circ}$, total internal reflection occurs and only evanescent waves can penetrate into the sol-gel layer. However, as the minimal thickness of the sol-gel layer is only $200 \mathrm{~nm}$, the evanescent waves can be partially coupled into the Si layers.

To circumvent the problems arising from the refractive index of sol-gel being lower than that of glass, we studied what would happen if the sol-gel was omitted and the nanotexture was directly carried by the glass. Figure 6 shows the results for unpolarized light, which we obtained by averaging the results for TE and TM 

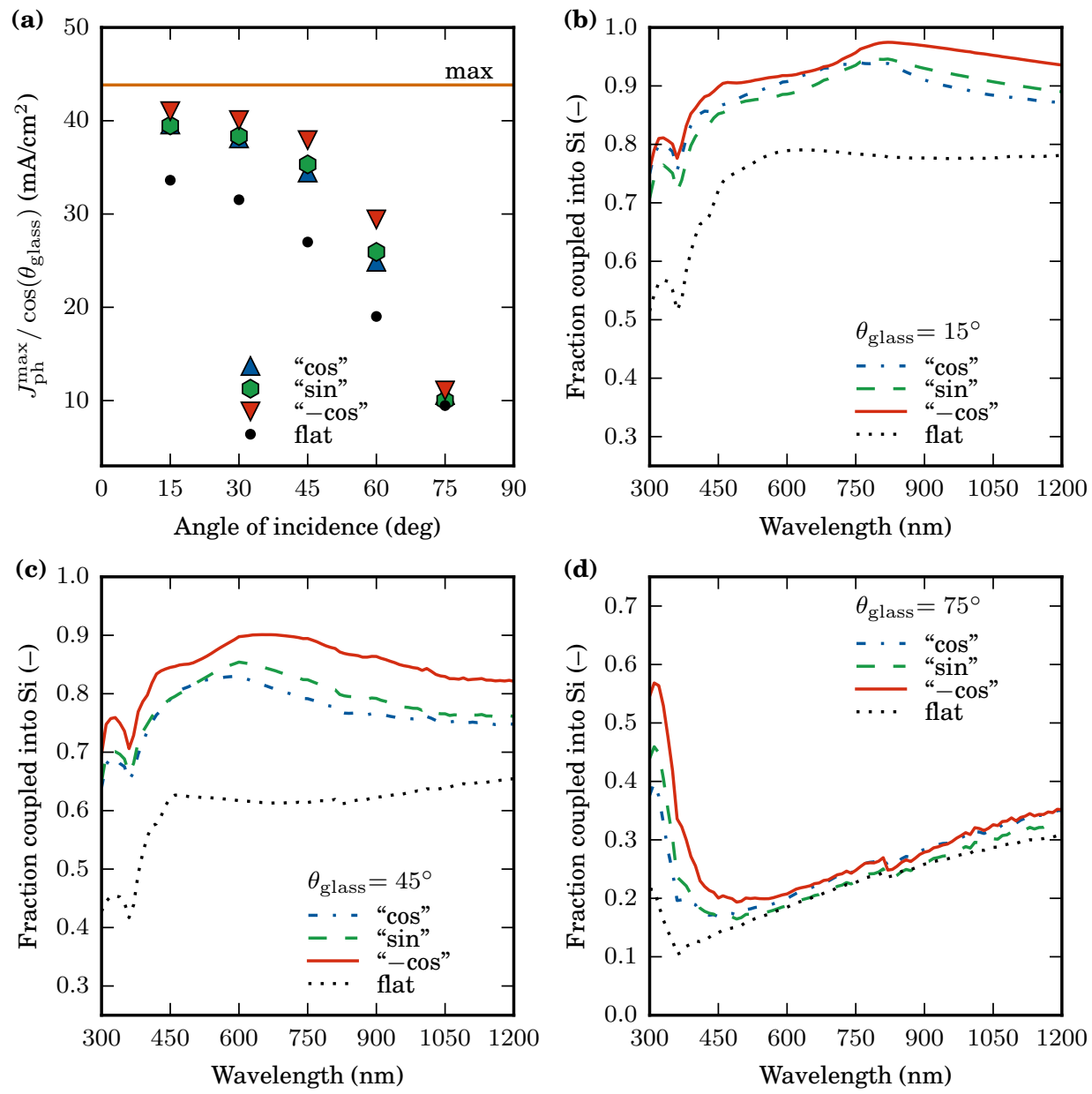

Figure 4. Results for oblique incidence of TE polarized light for the three geometries with $P=500 \mathrm{~nm}$ and $h=250 \mathrm{~nm}$ and a flat layer stack. In (a) the maximum achievable current density is shown while in (b)-(d) the spectra of the in-coupled light are shown for $\theta_{\text {glass }}=15^{\circ}, 45^{\circ}$ and $75^{\circ}$. 

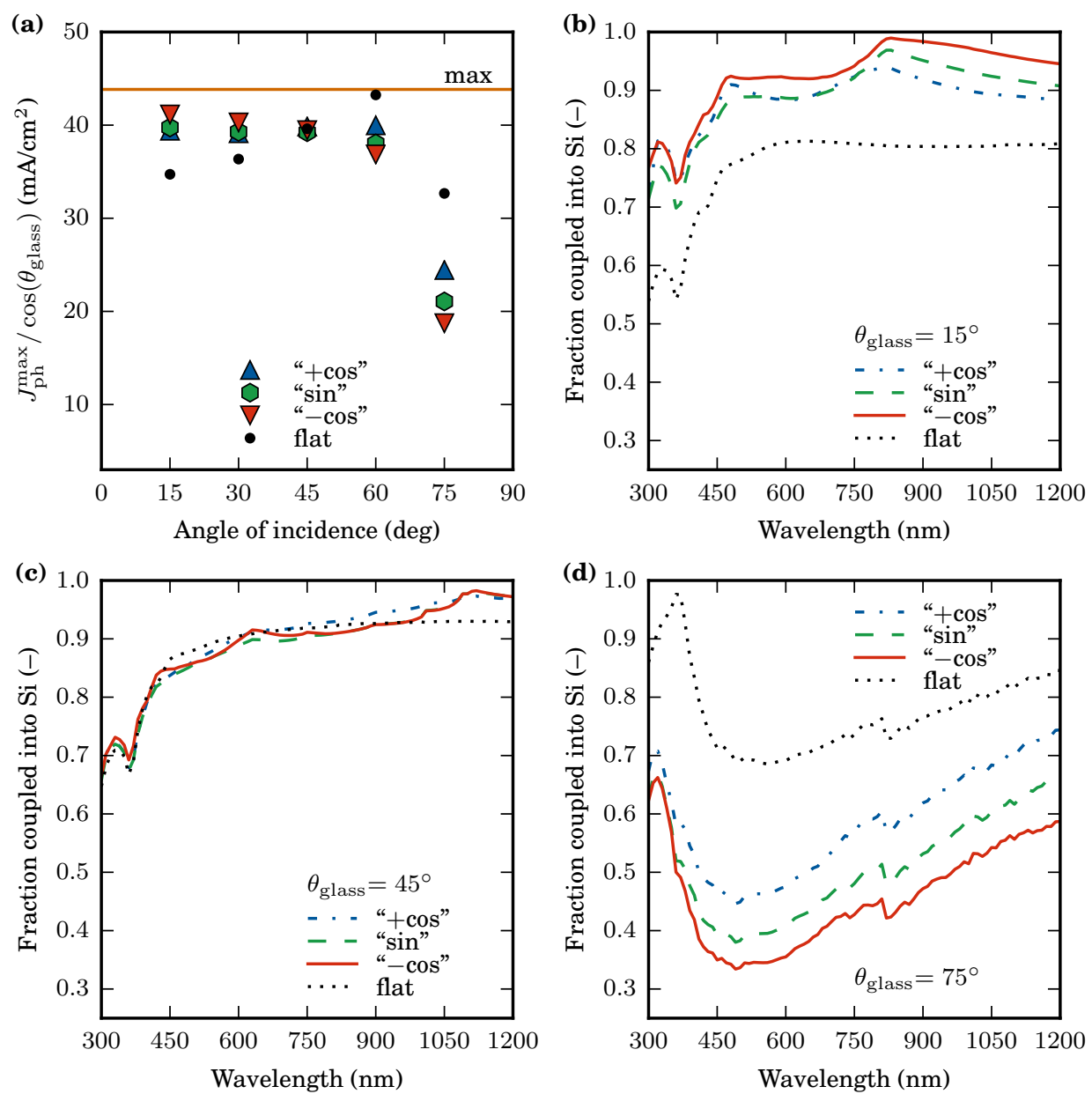

Figure 5. Results for oblique incidence of TM polarized light for the three geometries with $P=500 \mathrm{~nm}$ and $h=250 \mathrm{~nm}$ and a flat layer stack. In (a) the maximum achievable current density is shown while in (b)-(d) the spectra of the in-coupled light are shown for $\theta_{\text {glass }}=15^{\circ}, 45^{\circ}$ and $75^{\circ}$.
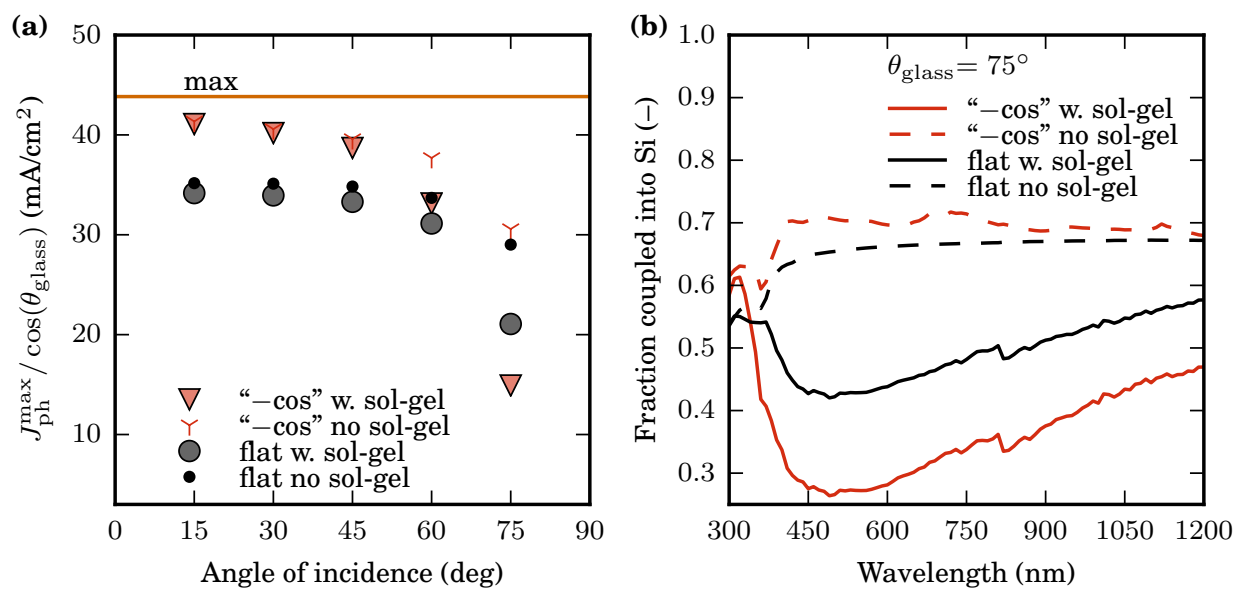

Figure 6. Results for oblique incidence of unpolarized light for layers stacks with and without the sol-gel layer. Both cases are shown for the negative cosine geometry with $P=500 \mathrm{~nm}$ and $h=250 \mathrm{~nm}$ and the flat case. In (a) the maximum achievable current density is shown while in (b) the spectra of the in-coupled light are shown for $\theta_{\text {glass }}=75^{\circ}$. 

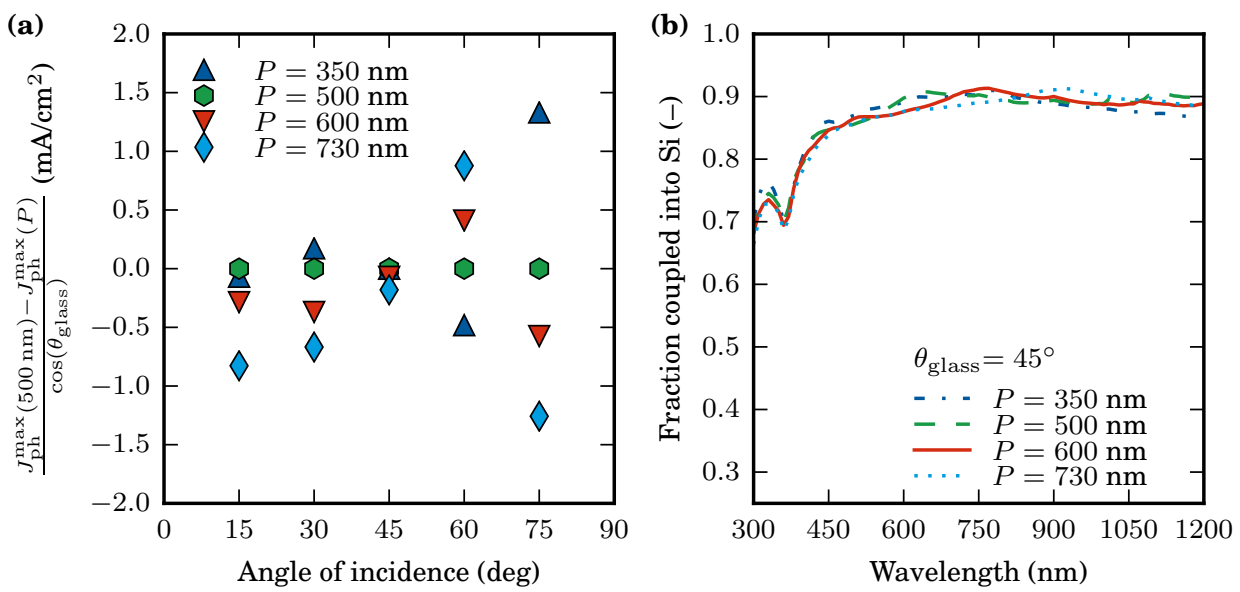

Figure 7. Results for oblique incidence of unpolarized light for the negative cosine texture for different periods $P$ and a structure height of $h=P / 2$ for the layer stack with sol-gel. In (a) the maximum achievable current density is shown while in (b) the spectra of the in-coupled light are shown for $\theta_{\text {glass }}=45^{\circ}$.

polarization. In Fig. 6 (a) $J_{\mathrm{ph}}^{\max } / \cos \theta_{\text {glass }}$ is shown. For small angles $<60^{\circ}$ the nanotextured layer stack outperforms the flat one, no matter whether the sol-gel is present or not. However, for the large angles $\left(60^{\circ}\right.$ and $75^{\circ}$ ) omitting the sol-gel has a clear advantage because of increasing reflection at the glass-sol-gel interface. At $\theta_{\text {glass }}=75^{\circ}$ the nanotextured stack outperforms the flat one when the sol-gel is omitted, which is not the case when the sol-gel is present. In Fig. 6(b) the fraction of the light coupled into the silicon absorber is shown for $\theta_{\text {glass }}=75^{\circ}$. We see that omitting the sol-gel layer strongly increases the in-coupled fraction across the whole spectrum.

Last, we study how strongly the period $P$ of the nanotexture affects the performance under oblique incidence. Figure 7 shows the results for the negative cosine texture for unpolarized light. In Fig. 7 (a), the deviation from $J_{\mathrm{ph}}^{\max } / \cos \theta_{\text {glass }}(P=500 \mathrm{~nm})$ is shown. Just as for $\theta_{\text {glass }}=0^{\circ}$ [see Fig. 3(a)], $500 \mathrm{~nm}$ is also the optimal period for $15^{\circ}$ and $45^{\circ}$. For the other angles, other periods are slightly superior for coupling the light into the absorber. Which period is the best for the overall performance depends on many factors such as the orientation of the PV module and cannot be answered in general. As an example, Fig. 7(b) shows the fraction of light coupled into the absorber at $\theta_{\text {glass }}=45^{\circ}$. We observe that it is very comparable for all four studied periods.

\section{CONCLUSIONS}

We studied coupling of light through a glass-silicon interface using hexagonal sinusoidal nanotextures. The investigated layer stack consists of glass, sol-gel (which carries the nanotexture) and silicon. We showed that these nanotextures are well suited for coupling light through a glass-silicon interface for oblique incidence. However, for very large angles $>60^{\circ}$, the coupling may be strongly reduced because total internal reflection can occur at the glass-sol-gel interface. We demonstrated that this problem could be solved by transferring the nanotexture directly into the glass.

\section{ACKNOWLEDGMENTS}

We acknowledge Florian Ruske for helping us with the ellipsometry measurements required for obtaining refractive index data. The results were obtained at the Berlin Joint Lab for Optical Simulations for Energy Research (BerOSE) of Helmholtz-Zentrum Berlin für Materialien und Energie, Zuse Institute Berlin and Freie Universität Berlin. The German Federal Ministry of Education and Research is acknowledged for funding the research activities of the Nano-SIPPE group within the program NanoMatFutur (No. 03X5520) and for funding within program SolarNano (13N13164). Further we acknowledge support by the Einstein Foundation Berlin through ECMath within subprojects SE6 and OT5. We thank David Eisenhauer for the useful discussions. 


\section{REFERENCES}

1. C. Becker, D. Amkreutz, T. Sontheimer, V. Preidel, D. Lockau, J. Haschke, L. Jogschies, C. Klimm, J. Merkel, P. Plocica, S. Steffens, and B. Rech, "Polycrystalline silicon thin-film solar cells: Status and perspectives," Sol. Energ. Mat. Sol. C. 119, 112-123 (2013).

2. D. Amkreutz, J. Haschke, T. Häring, F. Ruske, and B. Rech, "Conversion efficiency and process stability improvement of electron beam crystallized thin film silicon solar cells on glass," Sol. Energ. Mat. Sol. C. 123, 13-16 (2014).

3. J. Haschke, D. Amkreutz, L. Korte, F. Ruske, and B. Rech, "Towards wafer quality crystalline silicon thin-film solar cells on glass," Sol. Energ. Mat. Sol. C. 128, 190-197 (2014).

4. C. Becker, V. Preidel, D. Amkreutz, J. Haschke, and B. Rech, "Double-side textured liquid phase crystallized silicon thin-film solar cells on imprinted glass," Sol. Energ. Mat. Sol. C. 135, 2-7 (2015).

5. V. Preidel, D. Amkreutz, J. Haschke, M. Wollgarten, B. Rech, and C. Becker, "Balance of optical, structural, and electrical properties of textured liquid phase crystallized Si solar cells," J. Appl. Phys. 117, 225306 (2015).

6. K. Jäger, C. Barth, M. Hammerschmidt, S. Herrmann, S. Burger, F. Schmidt, and C. Becker, "Simulations of sinusoidal nanotextures for coupling light into c-Si thin-film solar cells," Opt. Express 24, A569-A580 (2016).

7. A. J. Wolf, H. Hauser, V. Kübler, C. Walk, O. Höhn, and B. Bläsi, "Origination of nano- and microstructures on large areas by interference lithography," Microelectron. Eng. 98, $293-296$ (2012).

8. J. Pomplun, S. Burger, L. Zschiedrich, and F. Schmidt, "Adaptive finite element method for simulation of optical nano structures," Phys. Status Solidi B 244, 3419-3434 (2007).

9. S. Burger, L. Zschiedrich, J. Pomplun, S. Herrmann, and F. Schmidt, "Hp-finite element method for simulating light scattering from complex 3D structures," Proc. SPIE 9424, 94240Z (2015).

10. "IEC:60904-3: Photovoltaic devices - Part 3: Measurement principles for terrestrial photovoltaic (PV) solar devices with reference spectral irradiance data," (2008).

11. K. Sakoda, Optical Properties of Photonic Crystals (Springer, Berlin/Heidelberg, 2005).

12. C. Barth, J. Probst, S. Herrmann, M. Hammerschmidt, and C. Becker, "Numerical characterization of symmetry properties for photonic crystals with hexagonal lattice," Proc. SPIE 9885 (2016). 